\title{
Erratum to: Hidden burden of non-medical spending associated with inpatient care among the poor in Afghanistan
}

\author{
Mohammad Omar Mashal $^{1} \cdot$ Keiko Nakamura $^{1} \cdot$ Masashi Kizuki $^{2}$
}

Published online: 20 July 2017

(C) Swiss School of Public Health (SSPH+) 2017

\section{Erratum to: Int J Public Health (2016) 61:661-671 DOI 10.1007/s00038-016-0833-9}

Unfortunately, few numerical values were incorrectly published in the original version and they are corrected by this Erratum.

In the Abstract section, second paragraph should read as: ... The subjects were $\mathbf{5 4 9 0}$ individuals included in a nationally representative survey in 2010 .
In the Introduction section third paragraph should read as: ... In Afghanistan, the government's share in the total health-care expenditure is only $6 \%$ and $18 \%$ is funded by international donors (Ministry of Public Health Afghanistan 2011).

In the Result section third paragraph should read as: ... The total amount of payments ranged from 20 to $\mathbf{8 0 0 , 0 0 0}$ Afghani.

The correct form of Table 3 is displayed here.

The online version of the original article can be found under doi:10.1007/s00038-016-0833-9.

Keiko Nakamura

nakamura.ith@tmd.ac.jp

1 Department of Global Health Entrepreneurship, Division of Public Health, Graduate School of Medical and Dental Sciences, Tokyo Medical and Dental University, Yushima 1-5-45, Bunkyo-ku, Tokyo 113-8519, Japan

2 Department of Health Promotion, Division of Public Health, Graduate School of Medical and Dental Sciences, Tokyo Medical and Dental University, Tokyo, Japan 
Table 3 Distribution of inpatient care payment (in Afghani) during recent overnight stays in the hospital in the previous 12 months (Afghanistan 2010)

\begin{tabular}{|c|c|c|c|}
\hline & $N$ & $\%$ & Mean \\
\hline All & 5490 & 100.0 & \\
\hline Total payment & & & 11,091 \\
\hline $20-1000$ & 594 & 10.8 & \\
\hline $1001-3000$ & 1357 & 24.7 & \\
\hline $3001-5000$ & 927 & 16.9 & \\
\hline $5001-10,000$ & 1196 & 21.8 & \\
\hline $10,001-800,000$ & 1416 & 25.8 & \\
\hline Diagnosis & & & 1677 \\
\hline 0-1000 & 4286 & 78.1 & \\
\hline $1001-3000$ & 666 & 12.1 & \\
\hline $3001-5000$ & 232 & 4.2 & \\
\hline $5001-10,000$ & 181 & 3.3 & \\
\hline $10,001-300,000$ & 125 & 2.3 & \\
\hline Medicine & & & 5879 \\
\hline $0-1000$ & 1690 & 30.8 & \\
\hline $1001-3000$ & 1715 & 31.2 & \\
\hline $3001-5000$ & 727 & 13.2 & \\
\hline $5001-10,000$ & 791 & 14.4 & \\
\hline $10,001-500,000$ & 567 & 10.3 & \\
\hline Accommodation & & & 1585 \\
\hline $0-1000$ & 4008 & 73.0 & \\
\hline $1001-3000$ & 958 & 17.4 & \\
\hline $3001-5000$ & 265 & 4.8 & \\
\hline $5001-10,000$ & 172 & 3.1 & \\
\hline $10,001-250,000$ & 87 & 1.6 & \\
\hline Transportation & & & 1950 \\
\hline $0-1000$ & 3668 & 66.8 & \\
\hline $1001-3000$ & 1025 & 18.7 & \\
\hline $3001-5000$ & 410 & 7.5 & \\
\hline $5001-10,000$ & 272 & 5.0 & \\
\hline $10,001-250,000$ & 115 & 2.1 & \\
\hline
\end{tabular}

50 Afghani 1 USD during the time of survey, April 2010, WQ1 poorest, $W Q 5$ richest, $R Q 1$ most remote, $R Q 5$ least remote 